\title{
Un anti-oncogène membranaire impliqué dans le cancer colorectal
}

Les années 1980 ont vu des progrès considérables dans l'isolement et la caractérisation des oncogènes cellulaires, progrès couronnés l'année dernière par le prix Nobel de médecine. Il est probable que les années 1990 seront au moins autant marquées par le développement de notre connaissance des gènes suppresseurs de tumeurs aussi appelés anti-oncogènes. Actuellement, alors qu'il en existe vraisemblablement au moins une centaine, seuls quatre ont été clonés et ont, par des expériences de transfection, démontré leurs propriétés suppressives. Le premier, isolé en 1985 chez la drosophile et appelé $1(2) g l[1]$, n'a pas pour l'instant d'homologue connu chez l'homme. Le deuxième, le gène $R b-1$ fut cloné un an plus tard et a depuis été l'objet de très nombreuses recherches [2]. Le troisième est le gène TP53 qui code pour la protéine p53. Certaines formes mutées de ce gène se sont montrées capables de coopérer avec un gène $H$-ras muté pour promouvoir un phénotype tumoral. Cependant il est aujourd'hui établi que sa forme non mutée supprime la tumorigénicité de divers types cellulaires [3-5]. Enfin, le quatrième, le gène $R a p-1 / K r e v-1$, qui est structurellement proche de la famille ras [6], a été cloné l'année dernière sur la base de sa capacité à restreindre la tumorigénicité de cellules transfectées par un gène $K$-ras activé [7]. A la différence de ce dernier, le gène $R b-1$ et le gène TP53, dont les produits sont localisés dans le noyau, sont connus pour être mutés dans plusieurs types de tumeurs humaines.

Bien que nous ne possédions pas encore les arguments fournis par les expériences de transfection, il semble ment par l'équipe de B. Vogelstein, soit le premier membre d'une nouvelle classe de gènes suppresseurs de tumeurs [8]. Ce gène, dont le produit est très vraisemblablement membranaire, a été trouvé modifié dans une dizaine de tumeurs colorectales. Son clonage est le résultat d'une combinaison exemplaire de techniques utilisées en génétique réverse.

L'observation initiale, à la base de ce travail, provient des études cytogénétiques de M. Muleris et de B. Dutrillaux. Dès 1985 [9], ces chercheurs français observaient la perte fréquente d'un bras court de chromosome 17 et d'un chromosome 18 dans un groupe de cancers colorectaux. Ces pertes rappelaient celles du chromosome 13 dans le rétinoblastome et suggéraient ainsi pour la carcinogenèse colorectale un mécanisme comparable à celui initialement proposé par Knudson : les segments chromosomiques perdus seraient chacun porteur d'un gène suppresseur de tumeur. Dans les cellules cancéreuses, aucun des deux homologues de ces gènes ne serait fonctionnel, l'un parce qu'il a disparu avec la délétion chromosomique, l'autre parce qu'il a subi une altération structurale. Dans ce dernier cas cependant, l'altération serait de taille insuffisante pour être détectée par la cytogénétique.

Pour aller plus loin dans la démonstration de ce mécanisme, il faut donc rechercher dans le chromosome cytogénétiquement intact la présence d'altérations supplémentaires. Dans ce but, plusieurs approches sont possibles. La plus immédiate consiste à examiner des gènes déjà localisés sur les segments chromosomiques impliqués. Cette approche fut fructueuse pour le bras court du chromosome 17 puisqu'elle devait révéler l'altération fréquente du gène $T P 53[10,11]$. Elle n'aboutit cependant pas pour le chromosome 18. Une approche différente fut alors utilisée qui consiste non pas à rechercher le gène directement, mais à en préciser la localisation en étudiant un grand nombre de tumeurs avec des sondes RFLP localisées sur les segments impliqués. En sélectionnant des patients constitutionnellement hétérozygotes pour ces sondes, il est possible de déterminer la région chromosomique la plus fréquemment perdue, région où réside vraisemblablement le gène recherché. Une telle étude montra que cette région était localisée sur le bras long du chromosome 18.

$\mathrm{Au}$ cours de ces études, une sonde RFLP isolée par O. Delattre et identifiant le locus D18S8 devait montrer des phénomènes surprenants. Ainsi, parmi trois tumeurs qui présentaient des délétions sur le bras long du chromosome 18 tout en conservant les deux allèles de ce locus, deux avaient des délétions distales [12] alors que la troisième présentait des délétions proximales [13] par rapport à lui. Cette situation suggérait que le locus D18S8 pouvait être contenu à l'intérieur même du gène recherché, l'inactivation pouvant avoir lieu indifféremment par des délétions de la partie 3' ou 5' du gène. Cette supposition a gagné en crédibilité lorsqu'une tumeur révéla une délétion homozygote pour le locus D18S8. Elle en gagna encore lorsque l'étude d'une tumeur supplémentaire démontra, à l'intérieur même du locus D18S8, l'existence d'une mutation somatique ponctuelle sur un site Mspl, enzyme utilisée pour révéler le polymorphisme. Cette mutation était telle qu'elle faisait apparaître la séquence consensus d'un site accep- 
teur pour l'épissage d'un ARN messager. Cette observation pouvait expliquer l'inactivation du gène postulé alors même que la mutation se produisait dans une séquence de type Alu en dehors d'un cadre ouvert de lecture.

Cet ensemble d'arguments paraissant suffisant, une marche sur le chromosome fut entreprise à la recherche de régions codantes. Près de $350 \mathrm{~kb}$ furent clonés. Les régions du génome conservées dans la phylogénie furent repérées mais aucune, prise isolément, ne permit la détection d'ARN messagers soit sur Northern blots soit sur diverses banques d'ADNc. Aussi les sept fragments les mieux conservés à travers les espèces furent séquencés. Des arguments forts en faveur de l'existence de régions codantes sur trois d'entre eux furent obtenus. En utilisant la méthode PCR il fut même possible de montrer que les séquences de deux des exons potentiels étaient effectivement présentes et correctement épissées dans des préparations d'ARN provenant des différents organes ou lignées cellulaires. Cette approche permit une étude de l'activité transcriptionnelle du gène désormais appelé $D C C$ (deleted in colorectal carcinomas). Observée dans tous les organes testés à l'exception du foie, elle est maximale dans le cerveau et nettement mesurable dans l'épithélium colique normal. Des tumeurs de plusieurs origines l'expriment. Cependant, la grande majorité des tumeurs colorectales ont un taux d'expression du gène $D C C$ inférieur à $5 \%$ de celui de la muqueuse normale. Souvent ces tumeurs ne l'expriment plus du tout, fournissant ainsi un argument supplémentaire en faveur du caractère antioncogène du produit du gène $D C C$ Une combinaison de fragments portant des exons fut utilisée pour cribler des banques d'ADNc. Les différents clones identifiés à ce jour révèlent 2854 bases de la région 5' du transcrit mature et permettent d'établir la séquence des 750 premiers acides aminés. Cette partie N-terminale connue de la protéine présente une homologie avec des membres de la superfamille des immunoglobulines et plus particulièrement avec la protéine d'adhérence des cellules de la crête neurale $(\mathrm{N}$ CAM). Il est donc vraisemblable que cette protéine soit membranaire et impliquée dans des processus de reconnaissance cellulaire. Des expériences de Northern blot avec des ARN extraits de cerveau révèlent que les transcrits matures ont des tailles de 10 et $12 \mathrm{~kb}$. La majorité de ce gène nous reste donc pour l'instant incon- nue. Il s'étend très probablement sur plus d'un million de paires de bases. Bien que partiel, l'isolement de cet $\mathrm{ADNc}$ a permis de repérer par la technique de Southern de nouveaux réarrangements géniques. A la différence de 44 tissus normaux, 7 sur 94 tumeurs primaires ou lignées de cancer colorectal présentèrent des fragments inhabituels. De manière tout à fait surprenante, les fragments réarrangés provenaient toujours d'une altération du même fragment de $170 \mathrm{bp}$ situé dans une région non codante. Celle-ci comporte dans sa séquence une alternance de purines et de pyrimidines suggérant que, in vivo, cette région de l'ADN puisse adopter une conformation Z. L'altération était à chaque fois due à l'insertion d'un élément génétique qui résista à toute tentative de clonage et/ou d'amplification par PCR. Cette particularité suggère une structure qui pourrait interférer avec le processus normal de transcription.

Dans les pays occidentaux, les adénocarcinomes colorectaux sont responsables d'une des plus fortes mortalités par cancer. Les connaissances accumulées ces deux dernières années sur les altérations génétiques dont il est le siège en font aussi la tumeur solide humaine la mieux connue sur le plan fondamental. Avec $K$-ras et le

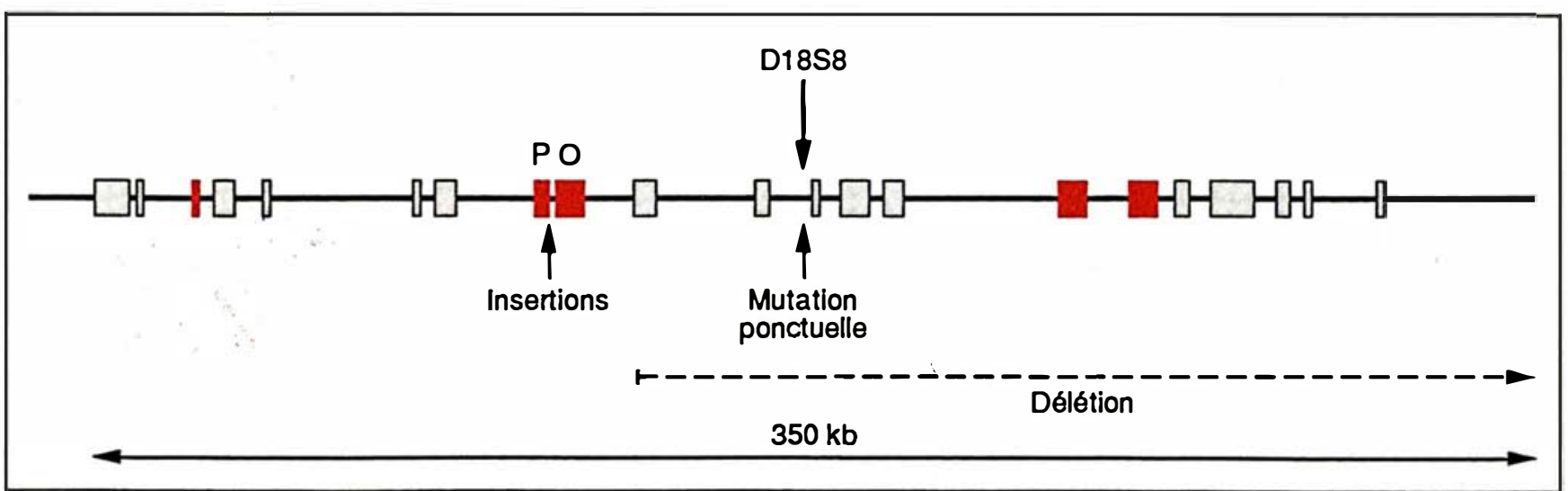

Figure 1. Éléments structuraux retrouvés dans la région clonée du gène DCC. La marche sur le chromosome entreprise par Fearon et al. a permis d'élargir la région du locus D18S8 sur $350 \mathrm{~kb}$. Dans cette région, les fragments EcoR1 conservés dans la phylogénie sont indiqués par des rectangles. Ceux contenant effectivement des exons du gène DCC sont rouges. Les exons contenus dans les fragments $P$ et $O$ furent utilisés pour l'étude de l'expression du gène par la méthode PCR. Les sites des altérations observées dans les cancers colorectaux sont indiqués sous le schéma du gène. La position du point de cassure de la délétion n'est pas connue avec précision. 
TP53, DCC constitue le troisième gène $y$ présentant des altérations récurrentes. L'isolement dans un proche avenir du gène $A P C$, responsable de la polypose adénomateuse rectocolique mais aussi impliqué dans les cancers colorectaux sporadiques, nous en fournira un quatrième. Il nous faudra alors comprendre le rôle de chacune de ces altérations et examiner leur éventuelle coopération dans la détermination du phénotype tumoral

Gilles Thomas

Chargé de recherche au Cnrs. Institut Curie, section de biologie, 26, rue d'Ulm, 75231 Paris Cedex 05, France

\section{RÉFÉRENCES}

1. Jacob L, Opper M, Metztoth B, Phannavong B, Mechler BM, Structure of the $1(2) g l$ gene of drosophila and delimitation of its suppressor domain. Cell 1987; 50: 215-25.

2. Cooper JA, Whyte P. RB and the cell cycle : entrance or exit ? Cell 1989; 58 : 1009-11.

3. Finlay CA, Hinds PW, Levine AJ. The p53 proto-oncogene can act as a suppressor of transformation. Cell 1989; 57 : 1083-96.

4. Eliyahu D, Michalovitz D, Eliyahu S, Pinhasi-Kimhi O, Oren M. Wild-type p53 can inhibit oncogene-mediated focus formation. Proc Nall Acad Si USA $1989 ; 86$ : 8763-93.

5. Hinds P, Finlay C, Levine AJ. Mutation is required to activate the $p 53$ gene for cooperation with the ras oncogene and transformation. J Virol 1989; 63 : 739-46

6. Pizaon V, Chardin P, Lerosey I, Olof son B, Tavitian A. Human cDNAs rap 1 and rap 2 homologous to the drosophila Dras3 encode proteins closely related to ras in the effector region. Oncogene $1988 ; 3: 201-4$.

7. Kitayama H, Sugimoto $Y$, Matsuzaki $T$, Ikawa $Y$, Noda M. A ras-related gene with transformation suppressor activity. Cell 1989 ; $56: 77-84$

8. Fearon ER, Cho KR, Nigro JM, et al. Identification of a chromosome $18 \mathrm{q}$ gene that is altered in colorectal cancers. Science 1990 ; 247: 49-56.

9. Muleris M, Salmon RJ, Zafrani B, Giro$\operatorname{det} \mathrm{J}$, Dutrillaux B. Consistent deficiencies of chromosome 18 and of the short arm of chromosome 17 in eleven of human large bowel cancer : a possible recessive mechanism. Ann Genet 1985 ; $28: 206-13$.

10. Baker SJ, Fearon ER, Nigro JM, et al. Chromosome 17 deletions and p53 mutations in colorectal carcinomas. Science $1989 ; 244$ : 217-21.

11. Nigro JM, Baker SJ, Presinger AC, et al. Mutations in the $p 53$ gene occur in diverse human tumour types. Nature $1989 ; 342$ : 705-8.

12. Vogelstein B, Fearon ER, Hamilton SR, el al. Genetic alterations during colorectaltumor development. $N$ Engl J Med 1988 ; 319 : 525-32.

13. Law D, Olschwang S, Monpezat JP, et al. Concerted monosyntenic loss in human colo- 